\title{
DIFFRACTION ON THE TWO-DIMENSIONAL SQUARE LATTICE*
}

\author{
H. S. BHAT ${ }^{\dagger}$ AND B. OSTING
}

\begin{abstract}
We solve the thin-slit diffraction problem for two-dimensional lattice waves. More precisely, for the discrete Helmholtz equation on the semi-infinite square lattice with data prescribed on the left boundary (the aperture), we use lattice Green's functions and a discrete Sommerfeld outgoing radiation condition to derive the exact solution everywhere in the lattice. The solution is a discrete convolution that can be evaluated in closed form for the wave number $k=2$. For other wave numbers, we give a recursive algorithm for computing the convolution kernel.
\end{abstract}

Key words. diffraction, discrete wave equation, lattice Green's functions, metamaterials, inductor-capacitor lattices, high-frequency analog circuits

AMS subject classifications. 78A45, 39A14, 39A60, 35J05

DOI. $10.1137 / 080735345$

1. Introduction. Consider the discrete wave equation on the semi-infinite square lattice with a Dirichlet condition along the line $i=0$ :

$$
\begin{aligned}
\frac{d^{2}}{d t^{2}} u_{i, j} & =c^{2}\left(\Delta_{d} u\right)_{i, j}, \quad i \geq 1, \\
u_{0, j}(t) & =f_{j} e^{\imath \omega t} .
\end{aligned}
$$

Here $f_{j}$ is supported only for $j \in \Sigma=[-A, A]$, where $A>0$ and $\Delta_{d}$ is the discrete Laplacian operator defined by

$$
\left(\Delta_{d} \phi\right)_{i j}=\phi_{i, j+1}+\phi_{i, j-1}+\phi_{i+1, j}+\phi_{i-1, j}-4 \phi_{i, j} .
$$

As a motivating experiment, we numerically solve (1.1) with initial conditions $u_{i, j}(0)=$ 0 and $\frac{d}{d t} u_{i, j}(0)=0$. We take a $400 \times 400$ lattice $(1 \leq i \leq 400,-199 \leq j \leq 200)$, $A=10$, constant data $f_{j}=1$, speed $c=1$, and the following successively larger frequencies: $\omega=\sqrt{1 / 2}, \omega=\sqrt{2}, \omega=\sqrt{11 / 4}$, and $\omega=\sqrt{7 / 2}$. In each case, we step forward in time from $t=0$ until some $t=T>0$ and then plot $\left|u_{100, j}(T)\right|$ for $-100 \leq j \leq 100$. Further details are given in Appendix A. The results of the numerical experiment, plotted in red $^{1}$ in Figure 1.1, show the diffraction of the spatially discrete waves that propagate from the aperture $\{0\} \times[-A, A]$ into the lattice.

The main result of this paper, which is what we used to plot the black curves in Figure 1.1, is a physical derivation of the exact solution (see (2.22) and Theorem

${ }^{*}$ Received by the editors September 15, 2008; accepted for publication (in revised form) August 6, 2009; published electronically November 13, 2009. This material is based upon work supported by the National Science Foundation (NSF) under grants DMS 07-53983 and DMS 06-02235, EMSW21RTG: Numerical mathematics for scientific computing. Any opinions, findings, and conclusions or recommendations expressed in this material are those of the author(s) and do not necessarily reflect the views of the National Science Foundation.

http://www.siam.org/journals/siap/70-5/73534.html

${ }^{\dagger}$ School of Natural Sciences, University of California, Merced, Merced, CA 95343 (hbhat@ ucmerced.edu).

$¥$ Applied Physics and Applied Mathematics, Columbia University, New York, NY 10027 (bro2103@columbia.edu).

${ }^{1}$ For interpretation of the references to color in this and all succeeding figures, the reader is referred to the electronic version of this article. 

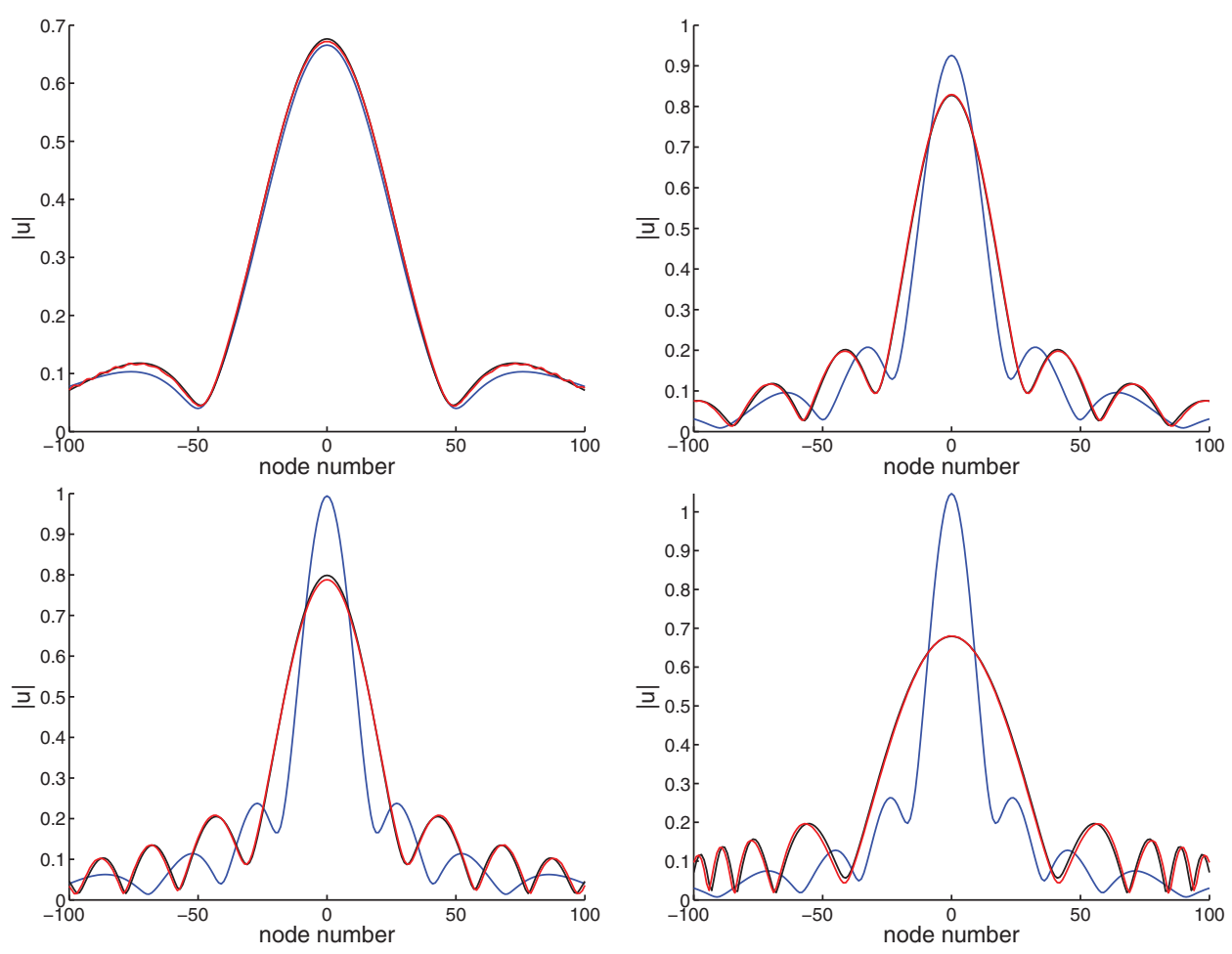

FIG. 1.1. From left to right, top to bottom, we have diffraction patterns for frequencies $\omega=$ $\sqrt{1 / 2}, \omega=\sqrt{2}, \omega=\sqrt{11 / 4}$, and $\omega=\sqrt{7 / 2}$. Results from the numerical experiment described in the text (time-stepping the discrete wave equation) are plotted in red. In blue we plot the results of continuum Rayleigh-Sommerfeld (R-S) diffraction theory, and in black we plot the results of our discrete diffraction theory. Note that continuum diffraction theory serves as a useful approximation of the red curve only for the smallest value of $\omega$, while the discrete diffraction theory closely tracks the red curve for all values of $\omega$ shown. (For interpretation of the references to color in this figure, the reader is referred to the electronic version of this article.)

2.1) of the discrete Helmholtz equation on the semi-infinite lattice with a Dirichlet condition on the left boundary:

$$
\begin{aligned}
\left(\Delta_{d}+k^{2}\right) U_{i j} & =0, \quad i \geq 1, \\
U_{0 j} & =f_{j} .
\end{aligned}
$$

Here $f_{j}$ is supported only for $j \in \Sigma$ for a finite set of integers $\Sigma$, and $U$ is required to satisfy an outgoing boundary condition specified in section 2.7. System (1.3) is a discrete version of the classical thin-slit diffraction problem; we use discrete versions of classical arguments to derive the solution. As shown in Figure 1.1, the solution of this discrete diffraction problem closely captures the behavior of the numerical experiment for all four values of $\omega$.

We have also plotted in blue the diffraction pattern predicted by standard R-S theory $[10,9]$ for a two-dimensional continuum:

$$
U(x, y)=-\frac{k x}{2 \imath} \int_{-A}^{A} u_{0}(\xi) \frac{H_{1}(k r)}{r} d \xi
$$

where $H_{1}$ is the Hankel function of the first kind and $r$ denotes the magnitude of 
the vector $\mathbf{r}$ that joins $(x, y)$ to $(0, \xi)$, a point on the aperture. As expected, the continuum theory diverges from the numerical experiment as $\omega$ increases.

Along the way to the solution, we apply recent asymptotic estimates of the lattice Green's function derived by Martin [24] to derive a discrete version of the Sommerfeld outgoing radiation condition. Having expressed the solution of (1.3) as a discrete convolution, we describe in detail a nontrivial computation of the convolution kernel. This involves applying different results from the literature to generate useable lattice Green's functions. For the particular wavenumber $k=2$, we are able to evaluate the convolution kernel in closed form. For $k \neq 2$, the numerical evaluation of the convolution solution of (1.3) is far faster than finding the steady-state solution to (1.1) through time-stepping.

1.1. Motivation. We are motivated by two application areas of recent interest: metamaterials and analog circuits. Of course, other applications exist, including massspring lattices and numerical discretizations of the continuum wave equation.

1.1.1. Left-handed two-dimensional inductor-capacitor metamaterial. Starting from the integer lattice $\mathbb{Z}^{2}$, suppose there is an inductor connecting each node $(i, j)$ to a common ground plane, and suppose that there is a capacitor connecting each node $(i, j)$ to its four nearest neighbors $(i \pm 1, j \pm 1)$. Assume that all inductances equal $L$ and all capacitances equal $C$, where both $L$ and $C$ are positive constants. In this case, Kirchhoff's laws of voltage and current imply the following second-order equation for the voltage $V_{i j}$ across the inductor at node $(i, j)$ :

$$
L C \frac{d^{2}}{d t^{2}}\left(\Delta_{d} V\right)_{i j}=V_{i j}
$$

This equation admits plane wave solutions $V_{i j}(t)=\exp \left[\imath\left(\omega t-\phi_{1} i-\phi_{2} j\right)\right]$ as long as the following dispersion relation is satisfied:

$$
\omega^{2}=\left[4 L C\left(\sin ^{2} \frac{\phi_{1}}{2}+\sin ^{2} \frac{\phi_{2}}{2}\right)\right]^{-1} .
$$

For both $m=1$ and $m=2$, we see that $\omega / \phi_{m}>0$ while $d \omega / d \phi_{m}<0$; for this reason, this type of inductor-capacitor lattice is referred to as a left-handed metamaterial [14]. Metamaterials have been of great recent theoretical and experimental interest $[28,18,23]$. Experimental groups have succeeded in fabricating artificial materials for which the dispersion relation is close to (1.5) for specific intervals of frequencies $\omega \in\left[\omega^{-}, \omega^{+}\right]$; see $[15]$, for example.

1.1.2. Standard two-dimensional inductor-capacitor lattice. In this system, there is a capacitor connecting each node $(i, j)$ to ground and inductors connecting each node to its nearest neighbors. Let $u_{i j}$ denote the voltage across the capacitor. Assuming all inductances equal $L$ and all capacitances equal $C$, Kirchhoff's laws of voltage and current can be used to derive (1.1a) with $c^{2}=(L C)^{-1}$ :

$$
L C \frac{d^{2}}{d t^{2}} u_{i j}=\left(\Delta_{d} u\right)_{i j}
$$

An inductor-capacitor lattice of this type was used to design a high-frequency power amplifier [2] on chip. Experimental measurements of the chip show that it generates $125 \mathrm{~mW}$ of power at $85 \mathrm{GHz}$, one of the best reported results at a frequency above $60 \mathrm{GHz}$ for any chip on a Silicon substrate [3]. The same lattice can be used in a 
different mode of operation as a Fourier transform device [1] or as an electrical prism [25]. Nonlinear versions of this lattice, where the capacitors are voltage-dependent, exhibit nonlinear constructive interference [5], which can be used to generate highpower, high-frequency harmonics of input signals [22].

1.2. Unified treatment via analysis of the discrete model. For the standard lattice, one is often interested in waves whose wavelength is large compared to the lattice spacing $h$. In this case, one may take the continuum limit of (1.6). The steps are standard: divide both sides of (1.6) by $h^{2}$, define the per-unit-length inductance and capacitance $\hat{L}=L / h$ and $\hat{C}=C / h$, and then take $h \rightarrow 0$. The discrete Laplacian $\Delta_{d}$ is replaced by the continuum Laplacian $\Delta$.

Note, however, that one cannot take the continuum limit of (1.4) in the same way — dividing both sides by $h^{2}$, defining $\hat{L}$ and $\hat{C}$ in the same way, and taking $h \rightarrow 0$ leads to blow-up on the right-hand side. Of course, we could have determined this from the dispersion relation (1.5) because $\omega \rightarrow \infty$ in the long-wave limit $\phi_{1}, \phi_{2} \rightarrow 0$.

Here we avoid continuum limits and instead directly analyze the discrete Helmholtz diffraction problem (1.3). This provides a unified treatment, valid when (1.1a) is either the standard discrete wave equation (1.6) or the left-handed discrete wave equation (1.4), and also valid for all waves, including waves with wavelength comparable to the lattice spacing $h$, which are of greatest interest in applications. Note that

- if we start from (1.1) as it is written and search for solutions of the form $u_{i j}(t)=e^{\imath \omega t} U_{i j}$, we obtain (1.3) with $k^{2}=\omega^{2} / c^{2}$, i.e., $k^{2}=\omega^{2} L C$;

- if we start from (1.1) where (1.1a) is replaced by (1.4) for $i \geq 1$ and search for solutions of the form $V_{i j}(t)=e^{\imath \omega t} V_{i j}$, we obtain (1.3) with $k^{2}=\left(\omega^{2} L C\right)^{-1}$. This shows that the solution of (1.3) can be used to solve propagation and diffraction problems for both standard and left-handed lattices. The solution can also be used to solve problems for composite right/left-handed (CRLH) [27] and dual-composite right/left-handed (d-CRLH) [13] lattices, where parallel and/or series LC blocks are used between nodes to bring about realistic dispersion relations that interpolate between (1.5) and the standard dispersion relation of (1.6). We have shown in past work [8] that in CRLH and d-CRLH lattices, voltages are governed by fourth-order discrete wave equations that reduce to (1.3a) if one assumes time-harmonic solutions.

Just as continuum diffraction theories may be derived starting from the continuum Helmholtz equation $\left(\Delta+k^{2}\right) U=0$, our diffraction theory proceeds from (1.3a). Before getting into the derivation, we review prior work on similar problems.

1.3. Prior work. The most relevant prior work is that of Shaban and Vainberg [30]; this paper solves the general propagation problem on a $d$-dimensional lattice with no boundaries. A detailed analysis proving that the discrete Sommerfeld condition singles out a unique solution of the problem is given. Together with the paper of Islami and Vainberg [20], this paper explains that, for example, as $t \rightarrow \infty$, the solution of the time-dependent problem (1.1) approaches $U e^{i \omega t}$, where $U$ solves (1.3). We should also mention the work of Schultz [29], who uses pointwise estimates of the Green's functions for the discrete wave equation to analyze the solution of both linear and nonlinear lattice wave equations in two and three spatial dimensions. Other papers from the numerical analysis literature $[4,32]$ analyze problems similar to (1.3) to determine how closely solutions of the discrete problem approximate solutions of the continuum problem.

While analytical considerations are very important, our goals in this paper are different. We provide, firstly, a physical derivation of the solution of the discrete diffraction problem (1.3). The arguments we use are discrete versions of arguments 
originally put forth by Sommerfeld. Our arguments are constructive and can be generalized to other lattice topologies, including the two-dimensional triangular and hexagonal lattices [7]. Secondly, we write the solution of (1.3) in a way that enables fast and accurate computation of discrete diffraction patterns. Despite their importance for practitioners who use lattices in electromagnetic and circuit applications, these considerations are absent from prior work on this topic. Both [30] and [20] do not describe the actual shape of discrete diffraction patterns and how they differ from continuum diffraction patterns with the same parameters.

2. Derivation of the discrete R-S theory. We proceed in stages, building up from basic identities to a discrete diffraction formula. The summation by parts and discrete Green's identity have appeared before [16, 31, 11]; we include our own derivations to keep this paper self-contained.

2.1. Summation by parts. The discrete version of integration by parts is

$$
\sum_{k=m}^{n} f_{k}\left(g_{k+1}-g_{k}\right)=f_{n} g_{n+1}-f_{m-1} g_{m}-\sum_{k=m}^{n} g_{k}\left(f_{k}-f_{k-1}\right),
$$

also referred to as Abel's lemma. This also gives

$$
\sum_{k=m}^{n} f_{k}\left(g_{k}-g_{k-1}\right)=f_{n+1} g_{n}-f_{m} g_{m-1}-\sum_{k=m}^{n} g_{k}\left(f_{k+1}-f_{k}\right) .
$$

Let $\partial_{d}^{2} g_{k}=g_{k+1}-2 g_{k}+g_{k-1}$ be the discrete one-dimensional second derivative operator. Then subtracting (2.2) from (2.1) yields

$$
\sum_{k=m}^{n} f_{k} \partial_{d}^{2} g_{k}=f_{n} g_{n+1}-f_{n+1} g_{n}+f_{m} g_{m-1}-f_{m-1} g_{m}+\sum_{k=m}^{n} g_{k} \partial_{d}^{2} f_{k} .
$$

2.2. Green's second identity. We associate the indices $i$ and $j$ with the horizontal and vertical directions, respectively. Let $\Omega$ be the rectangular region of the discrete lattice defined by $\Omega=\{(i, j) \mid W \leq i \leq E, S \leq j \leq N\}$, where $W, E, S, N \in \mathbb{Z}$. Then we claim that

$$
\begin{aligned}
\sum_{i j \in \Omega} U_{i j} \Delta_{d} V_{i j}-V_{i j} \Delta_{d} U_{i j} & =\sum_{W \leq i \leq E} U_{i N} V_{i N+1}-U_{i N+1} V_{i N}+U_{i S} V_{i S-1}-U_{i S-1} V_{i S} \\
+ & \sum_{S \leq j \leq N} U_{E j} V_{E+1 j}-U_{E+1 j} V_{E j}+U_{W j} V_{W-1 j}-U_{W-1 j} V_{W j} .
\end{aligned}
$$

Proof.

$$
\begin{aligned}
\sum_{i j \in \Omega} U_{i j} \Delta_{d} V_{i j}- & V_{i j} \Delta_{d} U_{i j}=\sum_{i=W}^{E} \sum_{j=S}^{N} U_{i j} \Delta_{d} V_{i j}-V_{i j} \Delta_{d} U_{i j} \\
= & \sum_{i=W}^{E}\left\{\sum_{j=S}^{N} U_{i j}\left(V_{i j+1}-2 V_{i j}+V_{i j-1}\right)-V_{i j}\left(U_{i j+1}-2 U_{i j}+U_{i j-1}\right)\right\} \\
& +\sum_{j=S}^{N}\left\{\sum_{i=W}^{E} U_{i j}\left(V_{i+1 j}-2 V_{i j}+V_{i-1 j}\right)-V_{i j}\left(U_{i+1 j}-2 U_{i j}+U_{i-1 j}\right)\right\} .
\end{aligned}
$$

Now apply (2.3) to each of the inner sums grouped inside curly braces. The result is precisely $(2.4)$. 
2.3. Remark. For $(i, j) \in \partial \Omega$, let $\delta_{\hat{\mathbf{n}}}$ denote the discrete derivative in the outward normal direction, so that on the four sides of the rectangle we have

$$
\begin{array}{lll}
\delta_{\hat{\mathbf{n}}} \phi_{i j}=\phi_{i N+1}-\phi_{i N}, & & \text { top, } \\
\delta_{\hat{\mathbf{n}}} \phi_{i j}=\phi_{E+1 j}-\phi_{E j}, & & \text { right, } \\
\delta_{\hat{\mathbf{n}}} \phi_{i j}=\phi_{i S-1}-\phi_{i S}, & & \text { bottom, } \\
\delta_{\hat{\mathbf{n}}} \phi_{i j}=\phi_{W-1 j}-\phi_{W j}, & & \text { left. }
\end{array}
$$

Let us return to (2.4) and consider the sum along the top side of the rectangle. It is easy to rewrite the sum using $\delta_{\hat{\mathbf{n}}}$ :

$$
\begin{aligned}
\sum_{W \leq i \leq E} U_{i N} V_{i N+1}-U_{i N+1} V_{i N} & =\sum_{W \leq i \leq E} U_{i N}\left(V_{i N+1}-V_{i N}\right)-\left(U_{i N+1}-U_{i N}\right) V_{i N} \\
& =\sum_{W \leq i \leq E} U_{i N} \delta_{\hat{\mathbf{n}}} V_{i N}-V_{i N} \delta_{\hat{\mathbf{n}}} U_{i N} .
\end{aligned}
$$

Carrying out the same procedure on all four sides, (2.4) can be summarized as

$$
\sum_{i j \in \Omega} U_{i j} \Delta_{d} V_{i j}-V_{i j} \Delta_{d} U_{i j}=\sum_{i j \in \partial \Omega} U_{i j} \delta_{\hat{\mathbf{n}}} V_{i j}-V_{i j} \delta_{\hat{\mathbf{n}}} U_{i j}
$$

which is of precisely the same form as the continuum version of Green's second identity.

2.4. Removing one point. Fix $(p, q) \in \Omega$. Let $\bar{U}_{p q}$ denote the average over the neighbors of $U_{p q}$ :

$$
\bar{U}_{p q}=\frac{1}{4}\left(U_{p q+1}+U_{p q-1}+U_{p+1 q}+U_{p-1 q}\right) .
$$

Then a direct calculation using (1.2) shows that

$$
-\left(U_{p q} \Delta_{d} V_{p q}-V_{p q} \Delta_{d} U_{p q}\right)=4 \bar{U}_{p q} V_{p q}-4 \bar{V}_{p q} U_{p q} .
$$

Let $\Omega_{0}=\Omega-(p, q)$ be the rectangle with the point $(p, q)$ removed. Then adding (2.6) to $(2.5)$ gives

$$
\sum_{i j \in \Omega_{0}} U_{i j} \Delta_{d} V_{i j}-V_{i j} \Delta_{d} U_{i j}=4 \bar{U}_{p q} V_{p q}-4 \bar{V}_{p q} U_{p q}+\sum_{i j \in \partial \Omega} U_{i j} \delta_{\hat{\mathbf{n}}} V_{i j}-V_{i j} \delta_{\hat{\mathbf{n}}} U_{i j} .
$$

Suppose $U$ and $V$ both satisfy (1.3a) in the region $\Omega_{0}$. Then

$$
\sum_{i j \in \Omega_{0}} U_{i j} \Delta_{d} V_{i j}-V_{i j} \Delta_{d} U_{i j}=0
$$

so the previous equation reduces to

$$
-4 \bar{U}_{p q} V_{p q}+4 \bar{V}_{p q} U_{p q}=\sum_{i j \in \partial \Omega} U_{i j} \delta_{\hat{\mathbf{n}}} V_{i j}-V_{i j} \delta_{\hat{\mathbf{n}}} U_{i j}
$$

Copyright $@$ by SIAM. Unauthorized reproduction of this article is prohibited. 
2.5. Lattice Green's function. Let $G_{i j ; p q}$ be the Green's function for (1.3a) centered at the point $(p, q)$, evaluated at $(i, j)$. By definition, $G_{i j ; p q}$ must satisfy

$$
\left(\Delta_{d}+k^{2}\right) G_{i j ; p q}=\delta_{i p} \delta_{j q}
$$

for all $(i, j) \in \mathbb{Z}^{2}$. The lattice Green's function $G_{i j ; p q}$ that satisfies (2.8) is quite well known $[21,17]$. Using trigonometric identities, one may write it in the form

$$
G_{i j ; p q}=\frac{1}{\pi^{2}} \int_{0}^{\pi} \int_{0}^{\pi} \frac{\cos [(i-p) \xi] \cos [(j-q) \eta]}{\sigma(\xi, \eta ; k)} d \xi d \eta
$$

with

$$
\sigma(\xi, \eta ; k)=k^{2}-4 \sin ^{2} \frac{1}{2} \xi-4 \sin ^{2} \frac{1}{2} \eta .
$$

From (2.9), it is evident that the lattice Green's function centered at $(p, q)$ evaluated at $(i, j)$ is the same as the lattice Green's function centered at $(0,0)$ evaluated at $(i-p, j-q)$, i.e.,

$$
G_{i j ; p q}=G_{i-p, j-q ; 00} .
$$

Henceforth, we use $G_{i-p, j-q}$ to denote $G_{i-p, j-q ; 00}$, i.e., if we do not specify otherwise, $G$ denotes the lattice Green's function centered at $(0,0)$.

2.6. Diffraction. Assume that $U$ solves (1.3a) in $\Omega$. By definition, $G_{i-p, j-q}$ solves (1.3a) in the punctured rectangle $\Omega_{0}$. Therefore, in (2.7), we can replace $V_{i j}$ by $G_{i-p, j-q}$. Note that $V_{p q}$ is then replaced by $G_{00}$. The left-hand side of (2.7) reduces to

$$
4 U_{p q} \bar{G}_{00}-4 G_{00} \bar{U}_{p q}=U_{p q}\left(\left(4-k^{2}\right) G_{00}+1\right)-G_{00}\left(4-k^{2}\right) U_{p q}=U_{p q},
$$

so we obtain

$$
U_{p q}=\sum_{i j \in \partial \Omega} U_{i j} \delta_{\hat{\mathbf{n}}} G_{i-p, j-q}-G_{i-p, j-q} \delta_{\hat{\mathbf{n}}} U_{i j} .
$$

We consider $\Omega$ defined by $W=1, E=M>0, N=M>0$, and $S=-M+1<0$. Then the previous equation can be written $U_{p q}=S_{1}+S_{2}$, where

$$
S_{1}=\sum_{j=-M+1}^{M} U_{1 j} \delta_{\hat{\mathbf{n}}} G_{1-p, j-q}-G_{1-p, j-q} \delta_{\hat{\mathbf{n}}} U_{1 j}
$$

and

$$
\begin{aligned}
S_{2}= & \sum_{i=1}^{M} U_{i M} \delta_{\hat{\mathbf{n}}} G_{i-p, M-q}-G_{i-p, M-q} \delta_{\hat{\mathbf{n}}} U_{i M} \\
& +\sum_{j=-M+1}^{M} U_{M j} \delta_{\hat{\mathbf{n}}} G_{M-p, j-q}-G_{M-p, j-q} \delta_{\hat{\mathbf{n}}} U_{M j} \\
& +\sum_{i=1}^{M} U_{i,-M+1} \delta_{\hat{\mathbf{n}}} G_{i-p,-M+1-q}-G_{i-p,-M+1-q} \delta_{\hat{\mathbf{n}}} U_{i,-M+1}
\end{aligned}
$$

Copyright (c) by SIAM. Unauthorized reproduction of this article is prohibited. 
2.7. Discrete Sommerfeld outgoing radiation condition. Our goal now is to show that if $U$ satisfies a discrete Sommerfeld outgoing radiation condition [30], then $\lim _{M \rightarrow \infty} S_{2}=0$. First let us estimate the three sums on the right-hand side of $S_{2}$ by

$$
\begin{aligned}
S_{2} \leq & \max _{i \in[1, M]} M\left(U_{i M} \delta_{\hat{\mathbf{n}}} G_{i-p, M-q}-G_{i-p, M-q} \delta_{\hat{\mathbf{n}}} U_{i M}\right) \\
& +\max _{j \in[-M+1, M]} 2 M\left(U_{M j} \delta_{\hat{\mathbf{n}}} G_{M-p, j-q}-G_{M-p, j-q} \delta_{\hat{\mathbf{n}}} U_{M j}\right) \\
& +\max _{i \in[1, M]} M\left(U_{i,-M+1} \delta_{\hat{\mathbf{n}}} G_{i-p,-M+1-q}-G_{i-p,-M+1-q} \delta_{\hat{\mathbf{n}}} U_{i,-M+1}\right) .
\end{aligned}
$$

Consider a point $(m, n)$ on any of the three sides (top, right, bottom) of the rectangle included in $S_{2}$. In polar coordinates centered at $(0,0)$, we have $(m, n)=$ $(R \cos \alpha, R \sin \alpha)$. Along the sides of the rectangle, we see that $R \in[M, \sqrt{2} M]$ and $\alpha \in[-\pi / 2, \pi / 2]$. Then, using a stationary phase calculation, Martin obtains the asymptotic form of the Green's function

$$
G_{m n} \sim \frac{e^{\imath\left(m \xi_{0}(\alpha, k)+n \eta_{0}(\alpha, k)\right)}}{\sqrt{2 \pi R}} F(\alpha, k) \text { as } R \rightarrow \infty,
$$

where there are two cases [24]. In the first case, $0<k^{2}<4$,

$$
\begin{aligned}
F(\alpha, k) & =\frac{-e^{\imath \pi / 4}}{\sqrt{k}} \frac{\left[4-k^{2}\left(\cos ^{4} \theta_{0}(\alpha, k)+\sin ^{4} \theta_{0}(\alpha, k)\right)\right]^{1 / 4}}{\sqrt{\left(4-k^{2}\right)\left(2-k^{2} \sin ^{2} \theta_{0}(\alpha, k) \cos ^{2} \theta_{0}(\alpha, k)\right)}} \\
\theta_{0}(\alpha, k) & =\tan ^{-1} \sqrt{-\lambda(\alpha, k)+\sqrt{\lambda(\alpha, k)^{2}+\tan ^{2} \alpha}} \\
\lambda(\alpha, k) & =\frac{2\left(1-\tan ^{2} \alpha\right)}{4-k^{2}} \\
\xi_{0}(\alpha, k) & =2 \sin ^{-1}\left[\frac{k}{2} \cos \theta_{0}(\alpha, k)\right] \\
\eta_{0}(\alpha, k) & =2 \sin ^{-1}\left[\frac{k}{2} \sin \theta_{0}(\alpha, k)\right] .
\end{aligned}
$$

In the second case, $4<k^{2}<8$,

$$
\begin{aligned}
F(\alpha, k) & =\frac{e^{-\imath \pi / 4}}{\left(8-k^{2}\right)^{1 / 4}} \frac{\left[k^{2}-4+2\left(8-k^{2}\right) \sin ^{2} \theta_{0}(\alpha, k) \cos ^{2} \theta_{0}(\alpha, k)\right]^{1 / 4}}{\sqrt{\left(k^{2}-4\right)\left(2-\left(8-k^{2}\right) \sin ^{2} \theta_{0}(\alpha, k) \cos ^{2} \theta_{0}(\alpha, k)\right)}} \\
\theta_{0}(\alpha, k) & =\tan ^{-1} \sqrt{-\lambda(\alpha, k)+\sqrt{\lambda(\alpha, k)^{2}+\tan ^{2} \alpha}} \\
\lambda(\alpha, k) & =\frac{2\left(1-\tan ^{2} \alpha\right)}{k^{2}-4} \\
\xi_{0}(\alpha, k) & =2 \cos ^{-1}\left[\frac{1}{2} \sqrt{8-k^{2}} \cos \theta_{0}(\alpha, k)\right] \\
\eta_{0}(\alpha, k) & =2 \cos ^{-1}\left[\frac{1}{2} \sqrt{8-k^{2}} \sin \theta_{0}(\alpha, k)\right] .
\end{aligned}
$$

Using these results, let us treat the three sides in turn. 
2.7.1. Top side. Consider the quantity

$$
S_{i}^{T}=M\left(U_{i M} \delta_{\hat{\mathbf{n}}} G_{i M}-G_{i M} \delta_{\hat{\mathbf{n}}} U_{i M}\right),
$$

where $i \in[1, M]$. In this section, when we use $G_{i j}$, we mean $G_{i j ; p q}$, the lattice Green's function centered at $(p, q)$. Note that $S_{i}^{T}$ is associated with the point $(i, M)$, and this point is associated with the angle $\alpha=\tan ^{-1}(M / i)$. For the left endpoint $(1, M)$, the angle $\alpha$ approaches $\pi / 2$ as $M \rightarrow \infty$. For the right endpoint $(M, M)$, the angle $\alpha$ equals $\pi / 4$ for all $M$.

For any $\alpha \in[\pi / 4, \pi / 2]$, let $r=\cot \alpha$. Let $\lfloor r M\rfloor$ denote the greatest integer less than $r M$. Then the sequence $(\lfloor r M\rfloor, M)$ has angle $\alpha$ in the limit, where $M \rightarrow \infty$. Clearly, the sequence of points $(\lfloor r M\rfloor, M+1)$ also has angle $\alpha$ in the $M \rightarrow \infty$ limit. The reason we mention this is that the asymptotic form of $\delta_{\hat{\mathbf{n}}} G_{i M}$ will depend on $G_{i M}$ as well as $G_{i M+1}$. We think of the sequence $(\lfloor r M\rfloor, M)$ as a "discrete ray" of asymptotic angle $\alpha$.

Along this ray with $i=\lfloor r M\rfloor$, the asymptotic form of $G$ gives, as $M \rightarrow \infty$,

$$
S_{i}^{T} \sim \sqrt{M}\left[\left(e^{\imath \eta_{0}(\alpha, k)}-1\right)-\delta_{\hat{\mathbf{n}}}\right] U_{i M} \frac{e^{\imath\left([i-p] \xi_{0}(\alpha, k)+[M-q] \eta_{0}(\alpha, k)\right)}}{\sqrt{2 \pi \csc \alpha}} F(\alpha, k) .
$$

To obtain this expression, we used

$$
R=M \sqrt{\lfloor r M\rfloor^{2} / M^{2}+1} \rightarrow M \csc \alpha \text { as } M \rightarrow \infty .
$$

So, along the top side, the discrete Sommerfeld outgoing radiation condition is

$$
\sqrt{M}\left[U_{i M}\left(e^{\imath \eta_{0}(\alpha, k)}-1\right)-\delta_{\hat{\mathbf{n}}} U_{i M}\right] \rightarrow 0 \text { as } M \rightarrow \infty .
$$

Assuming this condition holds for all $\alpha \in[\pi / 4, \pi / 2]$ (or equivalently for each $i \in$ $[1, M])$ as $M \rightarrow \infty$, the first term on the right-hand side of (2.12) goes to zero as $M \rightarrow \infty$.

2.7.2. Right/bottom sides. Calculations completely analogous to those just presented lead us to discrete Sommerfeld outgoing radiation conditions on the right side of the rectangle of interest

$$
\sqrt{M}\left[U_{M j}\left(e^{i \xi_{0}(\alpha, k)}-1\right)-\delta_{\hat{\mathbf{n}}} U_{M j}\right] \rightarrow 0 \text { as } M \rightarrow \infty,
$$

as well as the bottom side of the rectangle of interest

$$
\sqrt{M}\left[U_{i,-M+1}\left(e^{-\imath \eta_{0}(\alpha, k)}-1\right)-\delta_{\hat{\mathbf{n}}} U_{i,-M+1}\right] \rightarrow 0 \text { as } M \rightarrow \infty .
$$

We give the calculations leading to these conditions in Appendix B.

2.8. Method of images. We have shown that if (2.13), (2.14), and (2.15) hold, then $S_{2}$ - defined in (2.11) - vanishes. Then

$$
\begin{aligned}
U_{p q}=S_{1} & =\sum_{j=-M+1}^{M} U_{1, j} G_{-p, j-q}-U_{1, j} G_{1-p, j-q}-G_{1-p, j-q} U_{0, j}+G_{1-p, j-q} U_{1, j} \\
& =\sum_{j=-M+1}^{M} U_{1, j} G_{0, j ; p, q}-G_{1, j ; p, q} U_{0, j} .
\end{aligned}
$$


We now use the method of images to further simplify (2.16). Consider the lattice Green's function centered about the point $(-p, q)$; by definition, this function satisfies

$$
\left(\Delta_{d}+k^{2}\right) G_{i, j ;-p, q}=\delta_{-p, i} \delta_{q, j}
$$

for all $(i, j) \in \mathbb{Z}^{2}$. For fixed $(p, q)$, define

$$
G_{i, j ; p, q}^{-}=G_{i, j ; p, q}-G_{i, j ;-p, q} \text { for all }(i, j) \in \Omega \text {. }
$$

Because $G_{i, j ; p, q}^{-}$satisfies (2.8) in $\Omega$ and (1.3a) in $\Omega_{0}$, we can replace $G$ in (2.16) by $G^{-}$. Then, because $G_{i, j ; p, q}^{-}$vanishes for $i=0$, the second term on the right-hand side of (2.16) vanishes, and we obtain

$$
U_{p q}=\sum_{j=-M+1}^{M}-G_{1, j ; p, q}^{-} U_{0 j} .
$$

Of course, by (1.3b), we know that $U_{0 j}=f_{j}$ and that $f_{j}$ is supported only for $j \in \Sigma$. Hence

$$
U_{p q}=\sum_{j \in \Sigma}-G_{1, j ; p, q}^{-} f_{j} .
$$

Note from (2.18) that the desired Green's function is

$$
G_{1, j ; p, q}^{-}=G_{1-p, j-q}-G_{1+p, j-q},
$$

which by symmetry reduces to

$$
G_{1, j ; p, q}^{-}=G_{p-1, q-j}-G_{p+1, q-j}=G_{p, q-j ; 1,0}-G_{p, q-j ;-1,0} .
$$

Therefore, for each value of $k$, it is sufficient to find $G$ such that $\left(\Delta_{d}+k^{2}\right) G$ gives +1 at $(1,0),-1$ at $(-1,0)$, and zero everywhere else.

2.9. Discrete R-S formula. Let us examine the behavior of our solution (2.18) on $p=0$. Using (2.19), we get

$$
\begin{aligned}
U_{0 q} & =\sum_{j \in \Sigma}-G_{1, j ; 0, q}^{-} f_{j} \\
& =\sum_{j \in \Sigma}\left(-G_{1, j-q}+G_{1, j-q}\right) f_{j}=0 .
\end{aligned}
$$

Obviously, this does not match the true boundary condition (1.3b). To remedy the situation, we redefine $U_{p q}$ on the boundary.

TheOREM 2.1. The discrete diffraction problem (1.3a) is solved exactly by

$$
U_{p q}= \begin{cases}\sum_{j \in \Sigma}-G_{1, j ; p, q}^{-} f_{j}, & p \geq 1, \\ f_{q}, & p=0 .\end{cases}
$$

Proof. There are two cases. First let us examine what happens for $p \geq 2$. Since $p-1 \geq 1$, we may use the top branch of (2.22) to compute $U_{p-1, q}$ and the other four values of $U$ upon which $\left(\Delta_{d}+k^{2}\right) U$ depends. This yields

$$
\left(\Delta_{d}+k^{2}\right) U_{p q}=\sum_{j \in \Sigma}-\delta_{1, p} \delta_{j, q} f_{j}+\delta_{1,-p} \delta_{j, q} f_{j}=0
$$

because both $\delta_{1, p}$ and $\delta_{1,-p}$ vanish for $p \geq 2$.

Copyright (c) by SIAM. Unauthorized reproduction of this article is prohibited. 
Now, when $p=1$, we have to use both the $p \geq 1$ and the $p=0$ branches of $(2.22)$ to calculate $\left(\Delta_{d}+k^{2}\right) U$. With this in mind, we obtain

$$
\begin{aligned}
\left(\Delta_{d}+k^{2}\right) U_{1 q} & =U_{0 q}+U_{2 q}+U_{1, q+1}+U_{1, q-1}-4 U_{1 q}+k^{2} U_{1 q} \\
& =f_{q}+\left(0+U_{2 q}+U_{1, q+1}+U_{1, q-1}-4 U_{1 q}+k^{2} U_{1 q}\right) \\
& =f_{q}+\left(\Delta_{d}+k^{2}\right)\left[\sum_{j \in \Sigma}-G_{1, j ; p, q} f_{j}+G_{1, j ;-p, q} f_{j}\right]_{p=1} \\
& =f_{q}+\sum_{j \in \Sigma}\left[-\delta_{1, p} \delta_{j, q} f_{j}+\delta_{1,-p} \delta_{j, q} f_{j}\right]_{p=1} \\
& =f_{q}+\sum_{j \in \Sigma}-\delta_{j, q} f_{j} \\
& =f_{q}-f_{q}=0 .
\end{aligned}
$$

In the above calculation, we used (2.21) to go from (2.23) to (2.24).

Since (2.22) solves (1.3a) exactly and also satisfies the boundary condition (1.3b), it is an exact solution of the discrete diffraction problem (1.3). We refer to (2.22) as the discrete R-S formula.

2.10. Convolution. We can now use the expression for $G$ given in (2.9) to write

$$
G_{i, j ; p, q}^{-}=\frac{2}{\pi^{2}} \int_{0}^{\pi} \int_{0}^{\pi} \frac{\cos [(j-q) \eta] \sin (i \xi) \sin (p \xi)}{\sigma(\xi, \eta ; k)} d \xi d \eta .
$$

Note that (2.26) depends on $j$ and $q$ only through $(q-j)$. Slightly abusing notation, we write $G_{i, j ; p, q}^{-}=G_{i ; p}^{-}(q-j)$. Next, note that $U_{0, j}$ is supported only for $j \in \Sigma$. Putting everything together, we write $(2.22)$ as a discrete convolution:

$$
U_{p q}= \begin{cases}-\left(G_{1 ; p}^{-} * f\right)[q], & p \geq 1 \\ f_{q}, & p=0\end{cases}
$$

3. Computing the lattice Green's function. The main task in numerically evaluating (2.22) is to compute the lattice Green's function. For large values of $m$ and $n$, the integrands in (2.9) and (2.26) both suffer from extremely rapid oscillations and a curve of singularities where $\sigma(\xi, \eta ; k)=0$. Fortunately, there is a way to compute $G_{m n}$ that does not use numerical quadrature.

3.1. Diagonal elements. Recent work by Martin [24] provides expressions for the diagonal elements of the lattice Green's function in terms of Legendre functions:

$$
G_{n, n}=\frac{(-1)^{n}}{2 \pi \imath} \begin{cases}Q_{n-1 / 2}(z)-\frac{\pi \imath}{2} P_{n-1 / 2}(z), & k^{2}<4, \\ Q_{n-1 / 2}(z)+\frac{\pi \imath}{2} P_{n-1 / 2}(z), & k^{2}>4,\end{cases}
$$

with $z=1-\left(4-k^{2}\right)^{2} / 8$. Since $Q_{n-1 / 2}(z)$ blows up at $z=1$, we cannot use the above expressions when $k^{2}=4$. We return to this point later.

3.2. Off-diagonal elements. Using 8-fold symmetry, we need only to compute the lattice Green's function $G(m, n)$ in one octant of the plane. To do this, we apply a set of recurrence relations due to Morita [26]. Other recursive approaches may be 
found in the physics literature [12, 21]. Morita's equations use the diagonal elements of the lattice Green's function to uniquely determine the remaining elements:

$$
G_{1,0}=\frac{1-\left(k^{2}-4\right) G_{0,0}}{4},
$$

$$
G_{m, n}= \begin{cases}\left(4-k^{2}\right) G_{m-1,0}-G_{m-2,0}-2 G_{m-1,1}, & n=0, \\ \left(4-k^{2}\right) G_{m-1, n}-G_{m-2, n}-G_{m-1, n+1}-G_{m-1, n-1}, & 0<n<m-1, \\ \frac{4-k^{2}}{2} G_{m-1, n}-G_{m-1, n-1}, & n=m-1 .\end{cases}
$$

For $k^{2} \neq 4$, we use (3.1) and (3.2) in turn and compute all values of $G_{1, j ; p, q}^{-}$needed to evaluate (2.22). We have developed Mathematica code [6] that uses recurrence relation (3.2) to compute the lattice Green's function G. The code then uses G and the discrete convolution (2.27) to compute the diffracted field $U$.

For $k^{2}=4$, a different approach is needed.

3.3. Green's function for $\boldsymbol{k}^{2}=4$. In this case, we note that the discrete Helmholtz operator simplifies to a sum of the nearest neighbor elements of $U$ :

$$
\left(\Delta_{d}+4\right) U_{p q}=U_{p+1, q}+U_{p-1, q}+U_{p, q+1}+U_{p, q-1}=4 \bar{U}_{p q} .
$$

Using this representation of $\Delta_{d}+4$, we find by inspection the lattice Green's function

$$
G_{m n}^{k^{2}=4}=\frac{1}{4}(-1)^{1+\max \{|m|,|n|\}}=\left(\begin{array}{ccccccccc}
\ddots & & \multicolumn{1}{c}{} & & \vdots & & & & \\
& \frac{1}{4} & \frac{1}{4} & \frac{1}{4} & \frac{1}{4} & \frac{1}{4} & \frac{1}{4} & \frac{1}{4} & \\
& \frac{1}{4} & -\frac{1}{4} & -\frac{1}{4} & -\frac{1}{4} & -\frac{1}{4} & -\frac{1}{4} & \frac{1}{4} & \\
& \frac{1}{4} & -\frac{1}{4} & \frac{1}{4} & \frac{1}{4} & \frac{1}{4} & -\frac{1}{4} & \frac{1}{4} & \\
\cdots & \frac{1}{4} & -\frac{1}{4} & \frac{1}{4} & -\frac{1}{4} & \frac{1}{4} & -\frac{1}{4} & \frac{1}{4} & \ldots \\
& \frac{1}{4} & -\frac{1}{4} & \frac{1}{4} & \frac{1}{4} & \frac{1}{4} & -\frac{1}{4} & \frac{1}{4} & \\
& \frac{1}{4} & -\frac{1}{4} & -\frac{1}{4} & -\frac{1}{4} & -\frac{1}{4} & -\frac{1}{4} & \frac{1}{4} & \\
& \frac{1}{4} & \frac{1}{4} & \frac{1}{4} & \frac{1}{4} & \frac{1}{4} & \frac{1}{4} & \frac{1}{4} & \\
. & & & & \vdots & & & & \ddots
\end{array}\right),
$$

consisting of a central $-1 / 4$ surrounded by concentric alternating $1 / 4$ and $-1 / 4$ rings. Note that, unlike the lattice Green's functions for $k^{2} \in(0,4)$ and $k^{2} \in(4,8)$, the above function does not decay as $R=\sqrt{m^{2}+n^{2}} \rightarrow \infty$. Define

$$
H_{i j}=\left\{\begin{array}{ll}
(-1)^{i} & (i+j) \text { even } \\
0 & (i+j) \text { odd }
\end{array}=\left(\begin{array}{cccccccccc}
\ddots & & & & \vdots & & & & \\
& -1 & 0 & -1 & 0 & -1 & 0 & -1 & \\
& 0 & 1 & 0 & 1 & 0 & 1 & 0 & \\
& -1 & 0 & -1 & 0 & -1 & 0 & -1 & \\
\cdots & 0 & 1 & 0 & 1 & 0 & 1 & 0 & \ldots \\
& -1 & 0 & -1 & 0 & -1 & 0 & -1 & \\
& 0 & 1 & 0 & 1 & 0 & 1 & 0 & \\
& -1 & 0 & -1 & 0 & -1 & 0 & -1 & \\
. & & & & \vdots & & & & \ddots
\end{array}\right) .\right.
$$


Both $H_{i, j}$ and $H_{i-1, j}$ are nontrivial, nondecaying solutions of the homogeneous discrete Helmholtz equation (1.3a) on all of $\mathbb{Z}^{2}$. For $k^{2}=4$ and for all $\alpha, \beta \in \mathbb{R}$, the functions $G_{i j}^{k^{2}=4}+\alpha H_{i j}+\beta H_{i-1, j}$ are valid lattice Green's functions.

The same can be said for the method of images Green's function $G^{-}$. Using the specific form of $G^{k^{2}=4}$ given by (3.4) and (2.20), we obtain

$$
G_{-1,0 ; m, n}^{-}=G_{m, n ;-1,0}-G_{m, n ; 1,0}=\left(\begin{array}{cccccccccc}
\ddots & & & & \vdots & & & & \\
& \frac{1}{2} & 0 & 0 & 0 & 0 & 0 & -\frac{1}{2} & \\
& 0 & -\frac{1}{2} & 0 & 0 & 0 & \frac{1}{2} & 0 & \\
& 0 & 0 & \frac{1}{2} & 0 & -\frac{1}{2} & 0 & 0 & \\
\cdots & 0 & 0 & 0 & 0 & 0 & 0 & 0 & \ldots \\
& 0 & 0 & \frac{1}{2} & 0 & -\frac{1}{2} & 0 & 0 & \\
& 0 & -\frac{1}{2} & 0 & 0 & 0 & \frac{1}{2} & 0 & \\
& \frac{1}{2} & 0 & 0 & 0 & 0 & 0 & -\frac{1}{2} & \\
. & & & & \vdots & & & & \ddots
\end{array}\right) .
$$

The diagonal and antidiagonal elements alternate between $+1 / 2$ and $-1 / 2$, but do not decay to zero. Again, we could add arbitrary multiples of $H_{i, j}$ and/or $H_{i-1, j}$ to $G_{-1,0 ; i, j}^{-}$without changing $\left(\Delta_{d}+4\right) G_{-1,0 ; i, j}^{-}$. In computations, when $k^{2}=4$, we use (3.6).

3.4. Discrete R-S solution: $\boldsymbol{k}^{\mathbf{2}}=\mathbf{4}$. Using the $k^{2}=4$ Green's function (3.6) together with the discrete R-S formula (2.22), we obtain in closed form the exact steady-state solution of (1.1) with $u_{0, j}(t)=e^{2 \imath t}$ for $|j| \leq A$ and $u_{0, j}(t)=0$ for $|j|>A$. For example, with a small aperture $A=2$ we obtain $u_{m, n}(t)=U_{m, n} e^{2 \imath t}$, where

$$
U_{m, n}=\left(\begin{array}{cccccc} 
& & & & & \\
& & & & 1 / 2 & \\
& & & -1 / 2 & 1 / 2 & \\
& & 1 / 2 & -1 / 2 & 1 / 2 & \\
& -1 / 2 & 1 / 2 & -1 / 2 & 1 / 2 & \\
1 & -1 / 2 & 1 / 2 & -1 / 2 & 1 / 2 & \\
1 & -1 & 1 / 2 & -1 / 2 & 0 & \\
1 & -1 & 1 & 0 & 0 & \ldots \\
1 & -1 & 1 / 2 & -1 / 2 & 0 & \\
1 & -1 / 2 & 1 / 2 & -1 / 2 & 1 / 2 & \\
& -1 / 2 & 1 / 2 & -1 / 2 & 1 / 2 & \\
& & 1 / 2 & -1 / 2 & 1 / 2 & \\
& & & -1 / 2 & 1 / 2 & \\
& & & & 1 / 2 & \\
& & & & & \ddots
\end{array}\right) .
$$

Let us label the left-most column of this solution as the $m=0$ column. Then for all $(m, n)$ with $m \geq 1$, we find that the sum over the nearest neighbors vanishes, i.e., $4 \bar{U}_{m, n}=0$. Then, by (3.3), we obtain

$$
\frac{d^{2}}{d t^{2}} U_{m, n} e^{2 \imath t}=\Delta_{d} U_{m, n} e^{2 \imath t}
$$

Copyright $\odot$ by SIAM. Unauthorized reproduction of this article is prohibited. 


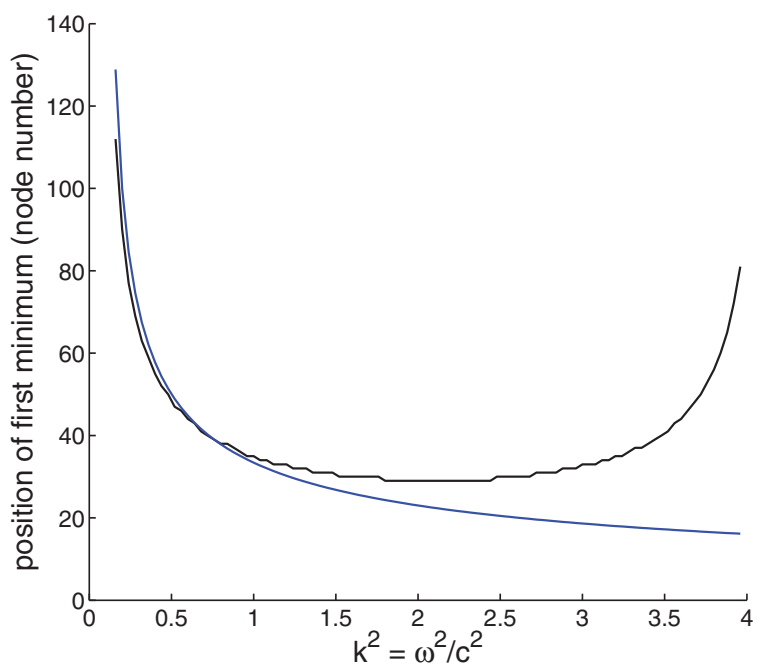

FIG. 4.1. Position of first minimum for the continuous (blue) and discrete (black) $R$-S theories for various values of $k^{2}=\omega^{2} / c^{2}$. (For interpretation of the references to color in this figure, the reader is referred to the electronic version of this article.)

so, as claimed, $u_{m, n}(t)$ gives an exact solution. The solution is steady-state in the sense that it is assumed that the driving force has been in existence since $t=-\infty$.

The solution for all other aperture sizes is similar: the initial wave splits into two traveling waves of half the amplitude moving at angles $+\pi / 4$ and $-\pi / 4$. The traveling waves are multiplied by -1 each time they move one unit to the right.

4. Conclusion. We opened this paper by describing a time-domain numerical experiment and proceeded to develop a discrete theory that exactly solves the steadystate version of the problem for all values of the driving frequency $\omega$. The question remains, For which values of $\omega$ does the continuum theory usefully predict the discrete result?

To address this question, we plot in Figure 4.1 the position of the first minimum of the diffraction pattern as predicted by the continuous R-S theory (blue) and discrete R-S theory (black) for various values of $k^{2}=\omega^{2} / c^{2}$ and constant amplitude input. For $k^{2} \lesssim 0.14$, both diffraction patterns have no minima. Above this value, the diffraction pattern looks qualitatively like that in Figure 1.1. The two diffraction theories closely agree for $k^{2} \lesssim 1$. The continuous R-S theory suggests that the diffraction pattern continues to condense about the $y$-axis as $k^{2} \rightarrow 4$. However, the discrete R-S theory predicts the first minimum moves away from the $y$-axis as $k^{2} \rightarrow 4$.

Since the discrete R-S formula (2.22) exactly solves (1.3) for all values of $k^{2}$, we can interpret Figure 4.1 as a measure of how well the continuous R-S theory agrees with the true diffraction pattern. Searching for solutions with wavevector $\overrightarrow{\mathbf{k}}=\left(k_{x}, k_{y}\right)=(\kappa, \kappa)$ in (1.1a), we obtain $\kappa=\cos ^{-1}\left[1-\omega^{2} /\left(4 c^{2}\right)\right] /(2 \pi)$. At $\omega^{2} / c^{2}=1$, $\kappa \approx(8.7)^{-1}$. According to Figure $4.1, \omega^{2} / c^{2}=1$ is where the discrete and continuum theories begin to diverge. Putting everything together, we see that if waves occupy less than nine lattice nodes, the continuous R-S theory no longer accurately predicts the output. In this case, the discrete R-S theory should be used.

Using similar lattice Green's function methods, discrete R-S theories can be derived for other regular lattices, such as the two-dimensional honeycomb, two-dimen- 
sional triangular, and three-dimensional cubic lattices. We expect that for all such media, discrete diffraction theories can answer questions regarding short-wavelength phenomena in situations where continuum diffraction theories cannot.

Appendix A. Numerical details. In section 1, when carrying out time-domain simulations of an $N \times N$ lattice, we began with

$$
\frac{d^{2}}{d t^{2}} \mathbf{u}=\Delta_{d} \mathbf{u}-\mathbf{b} \cdot \frac{d}{d t} \mathbf{u}
$$

The notation v.w denotes elementwise multiplication of equal-length vectors. For each $t, \mathbf{u}(t) \in \mathbb{R}^{N^{2}}$ is an ordinary column vector, with one unknown at each lattice node. If $A$ and $D$ are the $N^{2} \times N^{2}$ adjacency and degree matrices for the lattice, then $\Delta_{d}=A-D$. When $\Delta_{d} \mathbf{u}$ refers to a node on the left boundary, we simply use the value of $u$ on the boundary given by (1.1b).

Boundary conditions. To mimic a semi-infinite domain, we use first-order absorbing boundary conditions of Engquist-Majda [19] type on the top, right, and bottom boundaries. We accomplish this through the vector $\mathbf{b} \in \mathbb{R}^{N^{2}}$ in (A.1). For any node $j$ that borders the left boundary, where there is a Dirichlet condition, we set $b_{j}=0$. For all other nodes, we set $b_{j}=1$ if node $j$ is a noncorner boundary node, $b_{j}=2$ if node $j$ is a corner boundary node, and $b_{j}=0$ otherwise.

To see the effect of the damping term represented by $\mathbf{b}$, consider a noncorner boundary node on the right boundary of the square lattice. Then, rewriting (A.1) using two-dimensional indices, we obtain

$$
\frac{d^{2}}{d t^{2}} u_{m, n}=u_{m-1, n}+u_{m, n+1}+u_{m, n-1}-3 u_{m, n}-\frac{d}{d t} u_{m, n} .
$$

What if we instead had an infinite domain? Then, at the same boundary node $(m, n)$, we would instead have the equation

$$
\frac{d^{2}}{d t^{2}} u_{m, n}=u_{m+1, n}+u_{m-1, n}+u_{m, n+1}+u_{m, n-1}-4 u_{m, n} .
$$

The difference between the two equations is

$$
\left(u_{m, n}-u_{m+1, n}\right)-\frac{d}{d t} u_{m, n}=0,
$$

where the term in parentheses is a simple finite difference. Equation (A.4) is a firstorder, spatially discretized version of the Engquist-Majda absorbing boundary condition (ABC). Our lattice equation (A.1) is equivalent to the infinite lattice equation (A.3) plus the boundary condition (A.4) for nonforced, noncorner boundary nodes.

To see what happens at the corners, let us examine (A.1) in the upper-right corner. Again using two-dimensional indices, we obtain

$$
\frac{d^{2}}{d t^{2}} u_{m, n}=u_{m-1, n}+u_{m, n-1}-2 u_{m, n}-2 \frac{d}{d t} u_{m, n} .
$$

This equation follows from the infinite lattice equation (A.3) together with the spatially discrete $\mathrm{ABCs}$

$$
\begin{aligned}
& \left(u_{m, n}-u_{m+1, n}\right)-\frac{d}{d t} u_{m, n}=0, \\
& \left(u_{m, n}-u_{m, n+1}\right)-\frac{d}{d t} u_{m, n}=0 .
\end{aligned}
$$

The reason for setting $b_{j}=2$ for corner nodes should now be clear. 
We close by stating that, given how we have defined $\mathbf{b}$, a discrete $\mathrm{ABC}$ similar to (A.4) holds on the top, right, and bottom boundaries. A discrete ABC similar to (A.6) holds at the lower-right corner.

Physical interpretation. We may interpret (A.1) as a second-order equation for voltage $\mathbf{u}$ that can be derived from Kirchhoff's laws for an inductor-capacitor lattice as described in section 1.1.2. In this case, the $\mathbf{b}$ term arises from connecting nonforced boundary nodes to grounded resistors whose resistance equals the local lattice impedance $\sqrt{L / C}$. Our ABC amounts to impedance matching, a well-known concept in circuit design.

Time-stepping. Starting from (A.1), we discretize in time using centered differences. Let $\mathbf{u}^{k}$ denote our numerical approximation to $\mathbf{u}(k \Delta t)$. Let $B$ be a diagonal matrix with entries equal to the vector $\mathbf{b}$. Then our scheme is

$$
\left(I+\frac{\Delta t}{2} B\right) \mathbf{u}^{k+1}=2 \mathbf{u}^{k}-\mathbf{u}^{k-1}+(\Delta t)^{2}\left[\Delta_{d} \mathbf{u}^{k}\right]+\frac{\Delta t}{2} B \mathbf{u}^{k-1} .
$$

Since $I$ and $B$ are both diagonal, it is trivial to solve for $\mathbf{u}^{k+1}$ at each time step. To generate $\mathbf{u}^{1}$ given $\mathbf{u}^{0}$, we take one step using the standard semi-implicit Euler method applied to (A.1).

For $\omega=\sqrt{1 / 2}$, we choose $\Delta t=2 \pi /(128 \omega)$. For $\omega=\sqrt{2}$, we choose $\Delta t=$ $2 \pi /(256 \omega)$. For $\omega=\sqrt{11 / 4}$, we choose $\Delta t=2 \pi /(320 \omega)$. Finally, for $\omega=\sqrt{3 / 2}$, we choose $\Delta t=2 \pi /(384 \omega)$.

As this is a linear problem, it is easy to analyze the stability of the scheme by writing it as a map from $\left(\mathbf{u}^{k-1}, \mathbf{u}^{k}\right)$ to $\left(\mathbf{u}^{k}, \mathbf{u}^{k+1}\right)$. For all values of $\omega$ used in this paper, our time-step $\Delta t$ is chosen so that the eigenvalues of the mapping lie strictly inside the unit circle in the complex plane, ensuring stability.

Appendix B. Discrete Sommerfeld conditions. Our purpose here is to give conditions on $U$ under which the second and third terms on the right-hand side of (2.12) vanish. These terms correspond, respectively, to the right and bottom sides of the rectangle over which $S_{2}$ is summed in (2.11). The derivations here are completely analogous to that of section 2.7.1. As before, when we use $G_{i j}$, we mean $G_{i j ; p q}$, the lattice Green's function centered at $(p, q)$.

B.1. Right side. Consider the quantity

$$
S_{j}^{R}=2 M\left(U_{M j} \delta_{\hat{\mathbf{n}}} G_{M-p, j-q}-G_{M-p, j-q} \delta_{\hat{\mathbf{n}}} U_{M j}\right),
$$

where $j \in[-M+1, M]$. Note that $S_{j}^{R}$ is associated with the point $(M, j)$, and this point is associated with the angle $\alpha=\tan ^{-1}[j / M]$. For any $\alpha \in[-\pi / 4, \pi / 4]$, let $r=\tan \alpha$. Let $\lfloor\beta\rfloor$ denote the greatest integer less than or equal to $\beta$ if $\beta \geq 0$ or the smallest integer greater than $\beta$ if $\beta<0$. Then $-M+1 \leq\lfloor r M\rfloor \leq M$, and both sequences of points $(M,\lfloor r M\rfloor)$ and $(M+1,\lfloor r M\rfloor)$ have angle $\alpha$ in the $M \rightarrow \infty$ limit.

Along the ray with $j=\lfloor r M\rfloor$, the asymptotic form of $G$ gives, as $M \rightarrow \infty$,

$$
S_{j}^{R} \sim 2 \sqrt{M}\left[\left(e^{i \xi_{0}(\alpha, k)}-1\right)-\delta_{\hat{\mathbf{n}}}\right] U_{M j} \frac{e^{\imath\left([M-p] \xi_{0}(\alpha, k)+[j-q] \eta_{0}(\alpha, k)\right)}}{\sqrt{2 \pi \sec \alpha}} F(\alpha, k) .
$$

To obtain this expression, we used $R=M \sqrt{1+\lfloor r M\rfloor^{2} / M^{2}} \rightarrow M \sec \alpha$, again, as $M \rightarrow \infty$. This shows that, along the right side of the rectangle, the discrete Sommerfeld outgoing radiation condition is (2.14). Assuming this condition holds for all $\alpha \in[-\pi / 4, \pi / 4]$ (or, equivalently, for each $j \in[-M+1, M]$ ) as $M \rightarrow \infty$, the second term on the right-hand side of (2.12) goes to zero as $M \rightarrow \infty$.

Copyright $@$ by SIAM. Unauthorized reproduction of this article is prohibited. 
B.2. Bottom side. The treatment is nearly identical to the top side. Define

$$
S_{i}^{B}=M\left(U_{i,-M+1} \delta_{\hat{\mathbf{n}}} G_{i-p,-M+1-q}-G_{i-p,-M+1-q} \delta_{\hat{\mathbf{n}}} U_{i,-M+1}\right),
$$

where again $i \in[1, M]$. For any $\alpha \in[-\pi / 2,-\pi / 4]$, let $r=-\cot \alpha$. Let $\lfloor r M\rfloor$ denote the greatest integer less than $r M$. Both sequences $(\lfloor r M\rfloor,-M+1)$ and $(\lfloor r M\rfloor,-M)$ have angle $\alpha$ in the $M \rightarrow \infty$ limit.

Along the ray with $i=\lfloor r M\rfloor$, the asymptotic form of $G$ gives, as $M \rightarrow \infty$,

$$
S_{i}^{T} \sim \sqrt{M}\left[\left(e^{-\imath \eta_{0}(\alpha, k)}-1\right)-\delta_{\hat{\mathbf{n}}}\right] U_{i,-M+1} \frac{e^{\imath\left([i-p] \xi_{0}(\alpha, k)+[-M+1-q] \eta_{0}(\alpha, k)\right)}}{\sqrt{2 \pi \csc \alpha}} F(\alpha, k) .
$$

To obtain this expression, we used $R=M \sqrt{\lfloor r M\rfloor^{2} / M^{2}+(-M+1)^{2} / M^{2}} \rightarrow M \csc \alpha$. Therefore, along the bottom side, the discrete Sommerfeld outgoing radiation condition is (2.15). Again, assuming this condition holds for all $\alpha \in[-\pi / 2,-\pi / 4]$ (or, equivalently, for each $i \in[1, M])$ as $M \rightarrow \infty$, the third term on the right-hand side of (2.12) goes to zero as $M \rightarrow \infty$.

Acknowledgments. Finally, we thank the referees for their constructive criticism.

\section{REFERENCES}

[1] E. Afshari, H. S. Bhat, And A. Hajimiri, Ultrafast analog Fourier transform using twodimensional LC lattice, IEEE Trans. Circuits Syst. I. Regw. Pop., 55 (2008), pp. 2332 2343.

[2] E. Afshari, H. S. Bhat, A. Hajimiri, And J. E. Marsden, Extremely wideband signal shaping using one- and two-dimensional nonuniform nonlinear transmission lines, J. Appl. Phys., 99 (2006), p. 054901-1-16.

[3] E. Afshari, H. S. Bhat, X. Li, and A. Hajimiri, Electrical funnel: A new signal combining method, in Proceedings of the IEEE International Solid-State Circuits Conference (ISSCC'06), San Francisco, CA, 2006, pp. 206-208.

[4] A. Bamberger, J. C. Guillot, And P. Joly, Numerical diffraction by a uniform grid, SIAM J. Numer. Anal., 25 (1988), pp. 753-783.

[5] H. S. Bhat ANd E. Afshari, Nonlinear constructive interference in electrical lattices, Phys. Rev. E (3), 77 (2008), p. 066602-1-13.

[6] H. S. Bhat and B. Osting, DiscreteRS: Mathematica Code for evaluating the discrete Rayleigh-Sommerfeld convolution, http://www.cds.caltech.edu/ ${ }^{b}$ hat/discreteRS.nb.

[7] H. S. Bhat And B. Osting, Discrete Diffraction Theory for the $2 D$ Triangular Lattice, preprint, University of California, Merced, CA, 2008.

[8] H. S. BHAT AND B. Osting, Thin slit diffraction in conventional and dual composite right/lefthanded transmission line metamaterials, in Proceedings of the Asia-Pacific Microwaves Conference (APMC'08), Hong Kong and Macau, Wireless Communications Research Center at the City University of Hong Kong, December 2008.

[9] M. Born And E. Wolf, Principles of Optics, 6th ed. (corrected), Pergamon Press, Elmsford, NY, 1980.

[10] C. J. Bouwkamp, Diffraction theory, Rep. Progr. Phys., 17 (1954), pp. 35-100.

[11] S. BRLEK, The discrete Green theorem and some applications in discrete geometry, Theoret. Comput. Sci., 346 (2005), pp. 200-225.

[12] O. Buneman, Analytic inversion of the five-point Poisson operator, J. Comput. Phys., 8 (1971), pp. 500-505.

[13] C. CALOz, Dual composite right/left-handed (D-CRLH) transmission line metamaterial, IEEE Microw. Wireless Components Lett., 16 (2006), pp. 585-587.

[14] C. Caloz and T. Iтон, Electromagnetic Metamaterials, Transmission Line Theory and Microwave Applications, Wiley, Hoboken, NJ, 2006.

[15] C. Caloz and H. V. NGuyen, Novel broadband conventional- and dual-composite right/lefthanded (C/D-CRLH) metamaterials: Properties, implementation and double-band coupler application, Appl. Phys. A, 87 (2007), pp. 309-316.

Copyright (C) by SIAM. Unauthorized reproduction of this article is prohibited. 
[16] S. S. Cheng And R. F. Lu, Discrete Wirtinger's inequalities and conditions for partial difference equations, Fasc. Math., 23 (1991), pp. 9-24.

[17] E. N. Economou, Green's Functions in Quantum Physics, Springer-Verlag, New York, 2006.

[18] N. Engheta and R. W. Ziolkowski, Metamaterials: Physics and Engineering Explorations, IEEE Press, Piscataway, NJ, 2006.

[19] B. ENGQUist AND A. MAJDA, Absorbing boundary conditions for numerical simulation of waves, Proc. Natl. Acad. Sci. USA, 74 (1977), pp. 1765-1766.

[20] H. Islami And B. VainBerg, Large time behavior of solutions to difference wave operators, Comm. Partial Differential Equations, 31 (2006), pp. 397-416.

[21] S. Katsura AND S. InAwashiro, Lattice Green's functions for the rectangular and the square lattices at arbitrary points, J. Math. Phys., 12 (1971), pp. 1622-1630.

[22] G. N. Lilis, J. Park, W. Lee, G. Li, H. S. Bhat, and E. Afshari, Harmonic generation using nonlinear LC lattices, IEEE Trans. Microwave Theory and Techniques, submitted.

[23] R. Marqués, F. Martín, and M. Sorolla, Metamaterials with Negative Parameters: Theory, Design, and Microwave Applications, Wiley, Hoboken, NJ, 2008.

[24] P. A. Martin, Discrete scattering theory: Green's function for a square lattice, Wave Motion, 43 (2006), pp. 619-629.

[25] O. Momeni ANd E. Afshari, Electrical prism: A high quality factor filter for mm wave and terahertz frequencies, in Proceedings of the Asia-Pacific Microwaves Conference (APMC'08), Hong Kong and Macau, Wireless Communications Research Center at the City University of Hong Kong, December 2008.

[26] T. Morita, Useful procedure for computing the lattice Green's function-square, tetragonal, and bcc lattices, J. Math. Phys., 12 (1971), pp. 1744-1747.

[27] A. Sanada, C. Caloz, and T. Itoh, Characteristics of the composite right/left-handed transmission lines, IEEE Microw. Wireless Components Lett., 14 (2004), pp. 68-70.

[28] A. K. Sarychev and V. M. Shalaev, Electrodynamics of Metamaterials, World Scientific, Singapore, 2007.

[29] P. SchulTz, The wave equation on the lattice in two and three dimensions, Comm. Pure Appl. Math., 51 (1998), pp. 663-695.

[30] W. Shaban and B. Vainberg, Radiation conditions for the difference Schrödinger operators, Appl. Anal., 80 (2001), pp. 525-556.

[31] L. Yang and F. Albregtsen, Fast and exact computation of moments using discrete Green's theorem, in Proceedings of the Norwegian Image Processing and Pattern Recognition (NOBIM) Conference, 1994, pp. 82-90.

[32] A. ZEMLA, On the fundamental solutions for the difference Helmholtz operator, SIAM J. Numer. Anal., 32 (1995), pp. 560-570. 